\title{
La resolución de problemas en la prueba de Matemáticas de acceso a la universidad: procesos y errores
}

\section{Problem Solving in the Mathematics Section of the University Admission Tests: Processes and Mistakes}

\author{
Alberto Mallart Solaz \\ albert.mallart@ub.edu \\ Universidad de Barcelona, España
}

\begin{abstract}
Resumen:
La enseñanza de las Matemáticas ha ido cambiando a lo largo del tiempo como registran los currículos desde infantil hasta bachillerato. Pero la resolución de problemas siempre ha estado presente cobrando más importancia. A pesar de los cambios, las habilidades y capacidades Matemáticas no han mejorado. Por este motivo se ha analizado una muestra de 104 exámenes de la asignatura de Matemáticas de las Pruebas de Acceso a la Universidad de 2012. Se han estudiado las resoluciones con el propósito de detectar errores significativos y poder incidir en tales dificultades de aprendizaje. Se han analizado las resoluciones mecánicas, la ejecución con precisión de las operaciones, la aplicación con rigor de las propiedades y la capacidad creativa de resolución. Se ha observado una correcta asimilación de los aspectos mecánicos de las resoluciones pero una falta de creatividad en solucionar problemas geométricos, y falta de rigor y precisión en operaciones.
\end{abstract}

\section{Palabras clave:}

Evaluación; resolución de problemas; pruebas de acceso a la universidad; Matemáticas.

\begin{abstract}
:
Mathematics teaching has been changing over the last few years as is reflected in the curricula. But problem solving has been an increasingly important common factor. Despite changes, mathematical skills have not improved. This fact has motivated an analysis of 104 mathematics University Admission Tests in 2012. How students solved the six questions in the exam has been studied in order to detect meaningful mistakes and to try to correct the learning difficulties involved. Mechanical problem solving skills, precise execution of operations, the rigorous application of proprieties and the level of creativity involved in the solving of the problems have been analysed. The mechanical parts inherent in problem solving have been assimilated properly. However, creativity levels in solving geometric problems were not good, and operations were not completed thoroughly.
\end{abstract}

\section{Key words:}

Assessment; problem solving; university admission tests; mathematics. 
La resolución de problemas en la prueba de Matemáticas de acceso a la universidad: procesos y errores

Alberto Mallart Solaz

\section{Résumé:}

L'enseignement des mathématiques a évolué au cours du temps comme le démontrent les programmes officiels de la maternelle au lycée. Mais la résolution de problèmes a toujours été de plus en plus important. Malgré les changements, les aptitudes et les compétences en mathématiques ne se sont pas améliorées. Pour cette raison, nous avons analysé un échantillon de 104 examens du sujet de mathématiques à l'examen d'entrée à l'Université, 2012. Des résolutions ont été étudiées dans le but de détecter des anomalies significatives et d'influer sur les difficultés d'apprentissage. Nous avons analysé les résolutions mécaniques, l'exécution précise des opérations, I'application rigoureuse des propriétés et la capacité créative de la résolution. Il a été observé une bonne assimilation des aspects mécaniques des résolutions mais un manque de créativité dans la résolution de problèmes géométriques, et le manque de rigueur et de précision dans les opérations.

\section{Mots-clés:}

Evaluation ; résolution des problèmes; le test entrée à l'université; mathématiques.

Fecha de recepción: 17-7-2013

Fecha de aceptación: 1-12-2013

\section{Planteamiento del estudio}

El hecho de seleccionar los alumnos universitarios mediante un examen de ingreso en España fue una medida propugnada básicamente por Giner durante las dos últimas décadas del siglo XIX. La voluntad era dotar de seriedad a los estudios universitarios y de cierta homogeneidad a los estudiantes que ingresaban en las facultades. En la actualidad, las Pruebas de Acceso a la Universidad (PAU) contemplan dos tests matemáticos de estilos diferentes: Matemáticas (de carácter científico y tecnológico) y Matemáticas Aplicadas a las Ciencias Sociales.

En un estudio realizado por Corominas (2001) se identifican dos puntos del aprendizaje que inciden en el abandono o repetición de curso en estudios universitarios científico-tecnológicos: la inadaptación a las tareas de aprendizaje universitario y la falta de habilidades para estudiar. En el estudio de Riaguas y otros (2006) y en el de Boal y otros (2008) se evalúa el perfil del estudiante de nuevo acceso a grados de ingeniería concluyendo que existe una escasa autonomía como aprendiz y que tiene poco desarrollada la habilidad para aplicar y relacionar sus conocimientos matemáticos. Estos aspectos atañen al bagaje acumulado en conocimientos, procedimientos y actitudes. Por este motivo es interesante examinar el aprendizaje matemático y los errores que comete el estudiante cuando ha finalizado su preparación para ingresar en la universidad. 
Miralles y Deulofeu (2009) estudian las razones que llevan a los alumnos a una u otra elección de las cuestiones en la prueba de Matemáticas Aplicadas a las Ciencias Sociales (MACS) de las PAU y extraen conclusiones. Se plantean si ejerce alguna influencia el estilo gramatical del enunciado (corto y sintético o largo y poco directo), el tipo de contexto (matemático-formal o real-simulado), una resolución que requiere relacionar diversos apartados, una resolución que exige poseer recursos operativos sofisticados o cuestiones que permiten resoluciones a partir de diferentes razonamientos. También analizan aspectos curriculares de las cuestiones de las PAU como por ejemplo si alguna parte del temario está infrarrepresentada o si existen preguntas similares que seguramente se deben haber resuelto en el aula. Miralles y Deulofeu (2009) concluyen que los estudiantes eligen problemas de enunciados con términos lingüísticos sencillos aunque sean más difíciles. Además observan que los resultados obtenidos no guardan relación con la elección de lo que saben mejor. Constatan que el estudiante medio que accede a la asignatura de MACS en las PAU sabe poco más de lo que se le pregunta en la prueba.

Boal y otros (2008) estudian las habilidades Matemáticas evaluadas en las PAU motivados por los bajos índices de rendimiento en Matemáticas en las carreras universitarias de ingeniería y economía. Observan el tipo de alumnado que accede a estas carreras efectuando un "viaje educativo" en su formación matemática. Estudian la presencia de la resolución de problemas en las PAU procedentes de contextos fuera de los puramente matemáticos y afirman que la asignatura de MACS contiene muchos más que la de Matemáticas pues no se prepara tanto a los estudiantes para una futura abstracción. Boal y otros (2008) también estudian la penalización sobre la ortografía matemática en las PAU y comprueban que es poca. La comunicación matemática es otra característica que preocupa a Boal y otros (2008) llegando a determinar cómo la presencia de preguntas en las PAU en las que se requiere y valora el expresarse en lenguaje matemático aparece más en la asignatura de Matemáticas, aunque poco. Boal y otros (2008) concluyen que el enfoque del aprendizaje de las Matemáticas de bachillerato es un tanto superficial: aprenden a reproducir la información y técnicas para cumplir con los requisitos de las PAU.

A.Nortes y R.Nortes (2010) observan que a lo largo de la enseñanza primaria y secundaria la resolución de problemas está en todos los blo- 
La resolución de problemas en la prueba de Matemáticas de acceso a la universidad: procesos y errores

Alberto Mallart Solaz

ques de contenidos. Partiendo de una muestra de los exámenes de la asignatura de MACS de las PAU analizan las respuestas que dan a cada una de las cuestiones que constituyen la prueba y estudian los errores más significativos para contribuir a la mejora de la enseñanza y aprendizaje de la materia. Detectan que se hacen suposiciones iniciales que no están incluidas en el enunciado de los problemas. También observan que cuando los estudiantes no ven salida a su planteamiento, algunos no lo varían; incluso los hay que reiteran un procedimiento que conocen, sin poder pensar en otro más rápido. A.Nortes y R.Nortes (2010) registran que los alumnos prefieren contestar una cuestión sobre la que conocen el procedimiento de resolución que reflexionar sobre otro enunciado "nuevo" aunque sea más inmediato pero de resolución desconocida.

Ruiz de Gauna y otros (2011) estudian y clasifican los ejercicios propuestos en las PAU del País Vasco desde el 1994 hasta el 2008 en las asignaturas de Matemáticas y MACS. Han analizado su grado de dificultad y la relación con los resultados obtenidos, y también han examinado la percepción que tiene el profesorado sobre la dificultad de las partes de la prueba. Ruiz de Gauna y otros (2011) concluyen que el tipo de prueba influye en la metodología de enseñanza utilizada en el último curso de bachillerato y en la elaboración de los libros de texto; incluso en los estilos de enseñanza de los profesores. Para el análisis de los problemas que conforman la prueba se han fijado en características como: establecer problemas-tipo análogos en cuanto a su método de resolución, analizar cuáles de las partes de la prueba tienen una mayor diversidad de ejercicios y de resolución de problemas (según bloques de Álgebra, Geometría, Análisis de Funciones, Cálculo Integral, Probabilidad y Estadística), establecer en función de algunos criterios su nivel de dificultad (estándar y difícil) y relacionar éste con los resultados de la prueba.

Por otro lado, los informes PISA son evaluaciones internacionales que analizan las competencias que los alumnos alcanzan en la Secundaria. Desarrollar la competencia matemática en los escolares al término de la educación obligatoria se convierte en finalidad principal de la enseñanza y aprendizaje de las Matemáticas (Rico, 2007). Si bien los informes del 2003 y 2006 revelan que los jóvenes españoles han aprendido que las Matemáticas son un conjunto de reglas y técnicas que no saben cómo usar en su vida cotidiana, también se debe admitir que el informe del 2009 habla de una mejora paulatina en la capacidad de relacionar unas técnicas Matemáticas con otras incluso en contextos extraescolares. 
España se sitúa en la media de la OCDE (2006). En cuanto a las comunidades autónomas, PISA refleja que las diferencias entre unas y otras es a lo sumo de un 4\% (Instituto de Evaluación, 2010). El mérito tiene mucho que ver con el nuevo enfoque curricular de las Matemáticas: la resolución de problemas ha pasado a ser una metodología de enseñanza-aprendizaje donde se ha enfatizado el conectar el saber académico con las situaciones cotidianas.

Tomando conciencia de esta adaptación curricular promovida en parte por los informes PISA y teniendo presente los estudios realizados anteriormente comentados, a continuación se expone un nuevo estudio que se preocupa del razonamiento matemático que han adquirido los alumnos que ya han finalizado los estudios preuniversitarios. Se elige un contexto de suma relevancia como es el de las PAU y se toma una muestra de la asignatura de Matemáticas analizando los resultados y los errores.

\section{Método}

El objetivo principal de esta investigación es la detección de las diferentes dificultades que presentan los estudiantes preuniversitarios en la resolución de problemas. Más concretamente, se han propuesto los siguientes objetivos: a) analizar si se ha aprendido la parte mecánica de la resolución de problemas de Matemáticas; b) estudiar la capacidad creativa de resolución de problemas; c) analizar el grado de precisión con el que ejecutan las operaciones; d) estudiar el rigor con el que aplican las propiedades.

La muestra escogida de los alumnos que han sido preparados para acceder a carreras universitarias de ciencias está constituida por 104 estudiantes de Barcelona. Estos estudiantes han cursado la asignatura de Matemáticas en diversos centros públicos $(46,2 \%)$ y concertados $(53,8 \%)$ y pertenecen a diferentes niveles socioeconómicos.

Las PAU se convocan en Junio y Septiembre en cada Comunidad Autónoma. La asignatura de Matemáticas se estudia en primero y segundo de bachillerato en la modalidad de ciencias y tecnologías, y posibilita a los alumnos inscribirse en carreras universitarias de ciencias como Matemáticas, Físicas, Ingenierías. 
La resolución de problemas en la prueba de Matemáticas de acceso a la universidad: procesos y errores

Alberto Mallart Solaz

En las convocatorias de Matemáticas de las PAU de Cataluña se proponen 6 cuestiones de entre las cuales, el alumno debe escoger 5. En el examen se le pide que explique siempre qué quiere hacer y porqué. Cada cuestión vale 2 puntos. Se le permite el uso de calculadora siempre que no contenga información almacenada ni tampoco pueda recibirla.

Se valora que los alumnos aprendan a razonar y no únicamente resuelvan problemas tipificados. Por este motivo se procura que algunas cuestiones, sin tener ninguna dificultad especial, hagan pensar y no sean rutinarias. Se intenta saber hasta qué punto el alumno es capaz de resolver por sí mismo un problema para el cual dispone de los conocimientos suficientes pero no es una pregunta puramente metódica. También es importante que sepan leer e interpretar un enunciado. Debido a ello hay problemas de enunciado más largo.

Los criterios generales de evaluación insisten en que el alumno ha de explicar el porqué de sus respuestas. Incluso se puede valorar con un cero una cuestión con resultado correcto si el proceso resolutivo no se explica suficientemente. Las cuestiones que no estén resueltas completamente, se valorarán en función de las partes realizadas. En preguntas de carácter conceptual, el corrector intentará discernir si el alumno tiene claros los conceptos, aunque haya errores en la exposición. En ningún caso el corrector pondrá el acento en el rigor formal de las respuestas. Por regla general, las cuestiones no requieren cálculos largos. Por eso, el alumno debe esforzarse en hacer correctamente los cálculos que haya. Los errores de cálculo se tienen en cuenta en la puntuación total, con una importancia relativa.

La evolución de las notas medias de los exámenes de las PAU de junio de la asignatura de Matemáticas de estos últimos años muestra que son mejorables:

Tabla 1. Notas medias de los exámenes de Matemáticas de las PAU de junio 2000-2011

\begin{tabular}{llllllllllll}
\hline 2000 & 2001 & 2002 & 2003 & 2004 & 2005 & 2006 & 2007 & 2008 & 2009 & 2010 & 2011 \\
\hline 5.37 & 6.87 & 4.85 & 3.86 & 5.25 & 6.54 & 6.89 & 4.72 & 5.38 & 5.28 & 5.57 & 5.59 \\
\hline
\end{tabular}

El examen correspondiente a Junio de 2012 es el siguiente: 
La resolución de problemas en la prueba de Matemáticas de acceso a la universidad:

procesos y errores

CUESTIÓN 1.

Para qué valores del parámetro $m$ los planos

$\prod_{1}: x-y+m z=1, \prod_{2}: x-y+z=m, \prod_{3}: m y+2 z=3$

tienen como intersección una recta. [2 puntos]

CUESTIÓN 2.a

Dadas la recta $y=3 x+b$ y la parábola $y=x^{2}$,

a)Calculad la abscisa del punto donde la recta tangente a la parábola es paralela a la recta dada. [1 punto]

b)Calculad el valor del parámetro $b$ para que la recta sea tangente a la parábola.[1 punto]

CUESTIÓN 3. ${ }^{\mathrm{a}}$

Dados el plano $\prod: x-y+2 z-5=0$ y la recta $r:\left\{\begin{array}{l}x+y+z=0 \\ 2 x-y+z=10\end{array}\right.$,

a)Calculad el punto de intersección entre el plano y la recta. [1 punto]

b)Encontrad la ecuación continua de la recta $s$ contenida en el plano

$\prod$, que es perpendicular a la recta $r$ y corta a la recta $r$. [1 punto]

\section{CUESTIÓN $4 .^{\text {a }}$}

Dadas las matrices $A=\left(\begin{array}{cc}3 & 2 \\ -1 & 1\end{array}\right), B=\left(\begin{array}{cc}1 & -2 \\ 1 & 3\end{array}\right)$,

a) Comprobad que se cumple la igualdad $(A+B)(A-B)=A^{2}-B^{2}$. [1 punto]

b) 2 Es cierta esta igualdad para cualquier pareja de matrices cuadradas

$A, B$ del mismo orden? Responded razonadamente utilizando las propiedades generales de las operaciones entre matrices, sin utilizar matrices $A, B$ concretas. [1 punto]

\section{CUESTIÓN 5 . $^{\mathrm{a}}$}

Un triángulo equilátero de vértices $A, B, C$ tiene los lados de $8 \mathrm{~cm}$. Situamos un punto $P$ sobre una de las alturas del triángulo, a una distancia $x$ de la base correspondiente.

a)Calculad la altura del triángulo de vértices $A, B, C$. [0,5 puntos]

b)Indicad la distancia del punto $\mathrm{P}$ a cada uno de los vértices (en función de $x$ ). [0,5 puntos]

c)Determinad el valor de $x$ para que la suma de los cuadrados de las distancias del punto $P$ a cada uno de los tres vértices sea mínima. [1 punto]

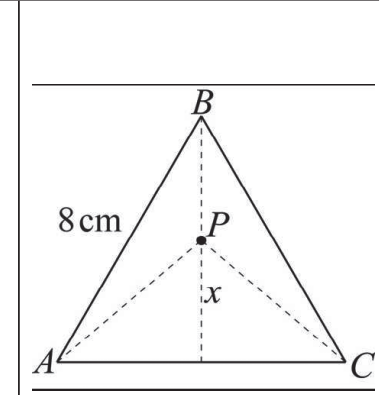


La resolución de problemas en la prueba de Matemáticas de acceso a la universidad: procesos y errores

Alberto Mallart Solaz

\section{CUESTIÓN 6 . $^{\mathrm{a}}$}

Dados los puntos $P=(1,0,0), Q=(0,2,0), R=(0,0,3), S=(1,2,3)$,

a)Calculad la ecuación cartesiana (es decir, de la forma $A x+B y+C z+D=0)$ ) del plano que contiene los puntos $P, Q, R$. [1 punto]

b)Comprobad si $P, Q, R, S$ son coplanarios (si están contenidos en un mismo plano). [1 punto]

\section{La muestra estudiada}

Los alumnos descartan una cuestión de las 6 presentadas. El porcentaje de los estudiantes que eligen cada cuestión se expone a continuación:

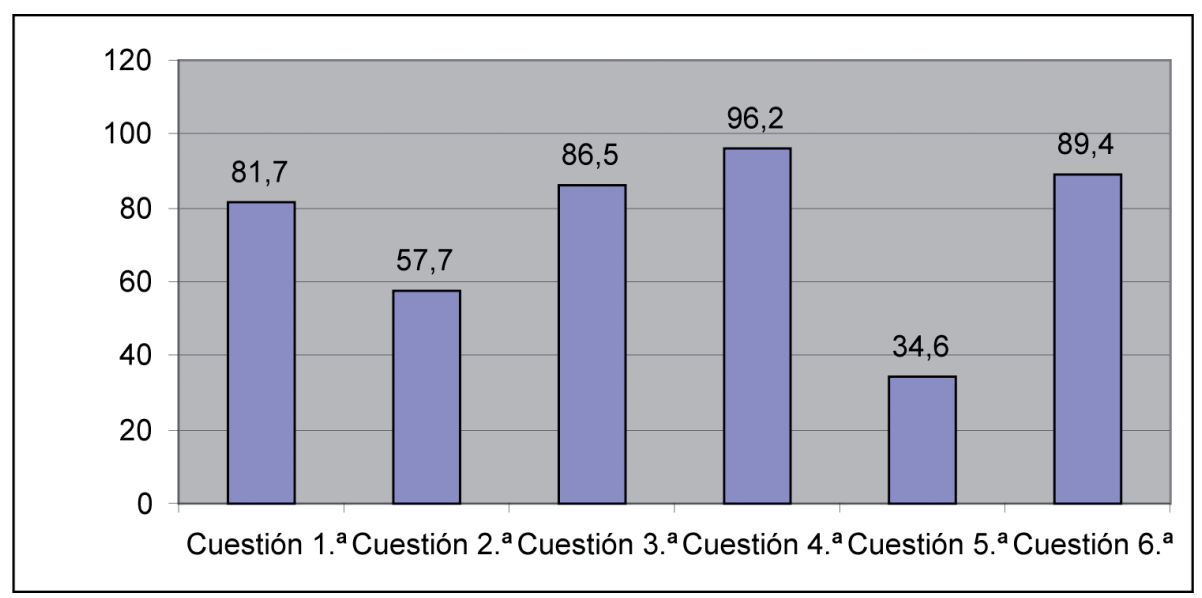

Figura 1. Porcentaje de la elección de las cuestiones

Por otro lado, el porcentaje de estudiantes que descarta cada cuestión o que no sabe resolverla se muestra en la tabla siguiente.

Tabla 2. Porcentajes de las cuestiones descartadas y nulas

\begin{tabular}{lllllll}
\hline CUESTIÓN & $1 .^{\mathrm{a}}$ & $2 .^{\mathrm{a}}$ & $3 .^{\mathrm{a}}$ & $4 .^{\mathrm{a}}$ & $5 .^{\mathrm{a}}$ & $6^{\mathrm{a}}$ \\
\hline Descartada & $18,3 \%$ & $42,3 \%$ & $13,5 \%$ & $3,8 \%$ & $65,4 \%$ & $10,6 \%$ \\
Puntuación nula & $16,3 \%$ & $6,7 \%$ & $35,6 \%$ & $3,8 \%$ & $5,8 \%$ & $14,4 \%$ \\
Descartada + Puntuación nula & $34,6 \%$ & $49,0 \%$ & $49,1 \%$ & $7,6 \%$ & $71,2 \%$ & $25,0 \%$ \\
\hline
\end{tabular}

A partir de la tabla se observa que la cuestión más descartada con un $65,4 \%$ ha sido la que trata de un triángulo equilátero y plantea preguntas 
de geometría plana, la número 5. La cuestión que menos alumnos han descartado con un $3,8 \%$ ha sido la que versa sobre álgebra matricial, la número 4. También es significativo el $42,3 \%$ que decide descartar a priori la segunda cuestión que trata de una recta, una parábola y sus tangentes respectivas. Aunque la tercera cuestión que es planteada a partir de una recta y un plano (geometría del espacio) sólo la rechazan un $13,5 \%$, sumando los que la han descartado con los que han obtenido una puntuación nula se ha conseguido el mismo $49 \%$ que en la segunda cuestión.

Todas las notas finales de los 104 exámenes se han redondeado en una escala que van de medio punto en medio punto, de 0 a 10. El rango de valores de las calificaciones finales obtenidas de esta muestra va desde la nota más baja (0) hasta la más alta (10). La nota media es 4,51 y la mediana es 4,5; la desviación típica es 2,723.

El número de estudiantes que se encuentra en el intervalo $(\bar{x}-\sigma, \bar{x}+\sigma)=(1,79,7,23)$ es de $64(61,5 \%)$, cerca de una distribución normal $(68,26 \%)$. El valor de la curtosis $(-0,975)$ indica que se concentra menos en torno a la media que una normal, y el valor de la asimetría $(0,184)$ indica una distribución ligeramente asimétrica a la derecha.

Tabla 3. Estadísticos descriptivos

\begin{tabular}{lllllll}
\hline \multirow{2}{*}{ Estadísticos } & Media & Mediana & Moda & Desv.Típica & Curtosis & Asimetría \\
\cline { 2 - 7 } & 4,51 & 4,5 & 2,5 & 2,723 & $-0,975$ & 0,184 \\
\hline
\end{tabular}

\section{Descripción de las resoluciones y de los errores más significativos}

Cada cuestión se puntúa desde cero hasta dos puntos. En la tabla 4 se expone un cuadro resumen de los resultados de cada cuestión, destacando las resoluciones totalmente incorrectas (" 0 ") y totalmente correctas ("2"), y la nota media. También se puede observar que: a) las cuestiones segunda, cuarta y sexta tienen una nota media aprobada; b) la cuestión sexta es la que recoge más puntuaciones máximas; c) la cuestión tercera es la que nota media más baja muestra, recogiendo también el mayor número de calificaciones nulas. 
La resolución de problemas en la prueba de Matemáticas de acceso a la universidad: procesos y errores

Alberto Mallart Solaz

Tabla 4. Características de los resultados de cada cuestión

\begin{tabular}{lllllll}
\hline CUESTIÓN & $1 .^{a}$ & $2 .^{a}$ & $3 .^{a}$ & $4 .^{a}$ & $5 .^{a}$ & $6 .^{a}$ \\
\hline Nota "0" & 17 & 7 & 37 & 4 & 6 & 15 \\
Nota "2" & 18 & 21 & 5 & 18 & 3 & 41 \\
Media & 0,86 & 1,16 & 0,57 & 1,11 & 0,82 & 1,34 \\
Desviación típica & 0,74 & 0,78 & 0,62 & 0,55 & 0,61 & 0,76 \\
Aprobados (\%) & 36,5 & 60 & 33,3 & 78 & 44,4 & 76,3 \\
\hline
\end{tabular}

A continuación se describen brevemente las diferentes características de las respuestas obtenidas cuestión por cuestión.

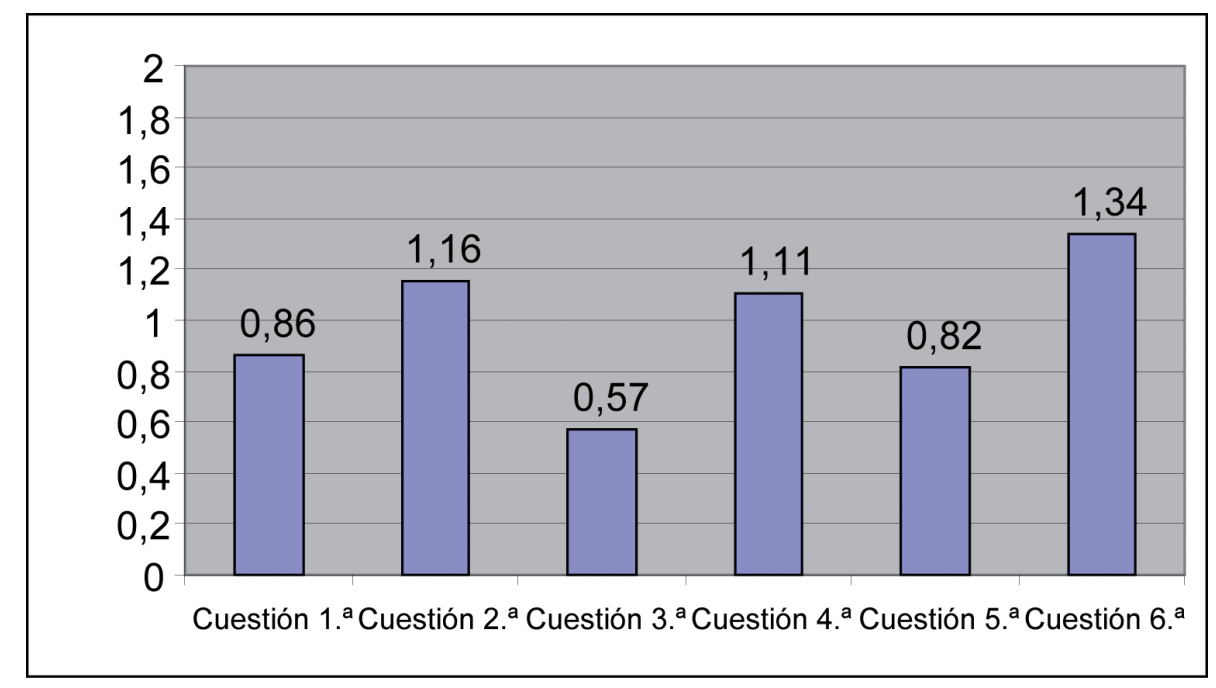

Figura 2. Nota media obtenida de cada cuestión

\section{Cuestión primera}

Dadas las ecuaciones de los tres planos, los estudiantes podían plantear la discusión en función del parámetro $m$ de dos maneras diferentes. Una manera consistía en plantear un sistema lineal de 3 ecuaciones con 3 incógnitas e imponer que fuera compatible indeterminado con 1 grado de libertad a partir del determinante de la matriz de coeficientes igualado a 0 (84\% alumnos). La otra manera era escalonando la matriz ampliada del sistema de ecuaciones (16\% alumnos). Estos porcentajes prueban lo incómodo que se sienten los estudiantes cuando han de hacer eliminación gaussiana donde aparecen parámetros. Además, la discusión con 
parámetros a partir del determinante consiste en resolver la ecuación al igualarlo a 0 , método más sencillo y más mecánico.

A continuación se muestra una clasificación con los errores más representativos:

- No han calculado bien el determinante de la matriz de coeficientes $(6,8 \%)$

- Aunque han encontrado correctamente los valores del parámetro para iniciar la discusión, no han sabido seguir (16,5\%)

- Después de haber encontrado los valores del parámetro correctos para discutir, han incurrido en errores graves de base teórica al extraer conclusiones (19,4\%). Por ejemplo: obtienen un sistema compatible determinado y afirman que los 3 planos se cortan en una recta; obtienen un sistema compatible indeterminado y afirman que los 3 planos no se cortan;...

Esta cuestión ha sido contestada por el 81,7\%; ha obtenido la puntuación máxima el $21,2 \%$ y el $20 \%$ ha obtenido una calificación nula por no haber sabido relacionar el hecho de que los tres planos se cortan en una recta con que el sistema formado por ellos sea compatible indeterminado. La puntuación media es de 0,86 y la desviación típica de 0,74 . Esta cuestión ha sido aprobada por un $36,5 \%$ de los que la contestaron.

\section{Cuestión segunda}

Los alumnos tenían que saber encontrar la pendiente de la recta $y=3 x+b$ para luego igualarla a la función derivada de la parábola $y=x^{2}$. Podían hacerlo de dos maneras: a) directamente a partir de la ecuación explícita de la recta (50,5\% alumnos); b) derivando la ecuación (12,1\% alumnos).

Se ha observado una gran cantidad de intentos infructuosos y de errores. Una clasificación representativa de ellos sería:

- Falta de comprensión del enunciado $(8,1 \%)$ que se refleja en resoluciones poco pertinentes: cálculo de integrales, cálculo del vértice de la parábola, invención incorrecta de rectas paralelas...

- No han sabido calcular la pendiente de la recta o la derivada de la ecuación de la recta $(10,1 \%)$

- No han igualado lo que debían, es decir, la derivada de la parábola con la pendiente de la recta $(12 \%)$. O bien han igualado la 
La resolución de problemas en la prueba de Matemáticas de acceso a la universidad: procesos y errores

Alberto Mallart Solaz

ecuación de la parábola con la ecuación de la recta, o bien han igualado la derivada de la parábola con la ecuación de la recta.

- Fijación con el origen $(7,1 \%)$ : han buscado la tangente a la parábola en el $(0,0)$, o han igualado la derivada de la parábola a 0 , o han substituido el $(0,0)$ en la ecuación de la recta para encontrar el valor de $b$

- Han cometido errores de cálculo de resolución de ecuaciones $(9,1 \%)$ :

${ }^{\circ}$ Igualando derivadas: $3=2 \mathrm{x} ; \mathrm{x}=2 / 3$

○ Calculando el parámetro $b: \frac{9}{4}=\frac{9}{2}+b ; b=\frac{9}{4}-\frac{9}{2}=\frac{-9}{2}$

Esta cuestión ha sido contestada por el 57,7\%; la puntuación máxima ha sido obtenida por el $35 \%$ y el $11,7 \%$ ha obtenido una calificación nula por no haber sabido encontrar las pendientes de la recta y de la parábola. La puntuación media es de 1,16 y la desviación típica de 0,78. Esta cuestión ha sido aprobada por un $60 \%$ de los que la contestaron.

\section{Cuestión tercera}

Existen diversas formas para calcular el punto de intersección entre el plano y la recta. Una manera sencilla sería resolver el sistema de 3 ecuaciones con 3 incógnitas formado por la ecuación del plano y las dos ecuaciones de la recta por el método de Gauss, por el método de Cramer o directamente resolviendo el sistema (37,8 \% alumnos). Aunque también podría resolverse encontrando las ecuaciones paramétricas de la recta $r$ y substituyéndolas en el plano $\Pi$ (17,4\% alumnos).

Los errores que han cometido los alumnos en esta cuestión han sido particularmente difíciles de tipificar tanto por su diversidad como por su significado. Las puntuaciones nulas o bajas no preocupan tanto por los errores de cálculo en resolver ecuaciones o en escalonar matrices (33\%) como por inventarse procesos resolutivos incoherentes (51,3\%). Preocupan los razonamientos teóricos seguidos para calcular el punto de intersección de la recta y el plano por ser absurdos e implicar una pérdida de tiempo considerable. Por ejemplo, el razonamiento erróneo más seguido ha sido la elección del plano $\{z=0\}$ para calcular la intersección con $r(10,5 \%)$. 
Ha habido menos alumnos que han Ilegado con éxito a resolver el segundo apartado. No han sabido hallar $\vec{v}_{\boldsymbol{r}}(41,7 \%)$, y de los que sí han sabido, sólo la mitad ha sabido calcular $\vec{v}_{S}$.

Resulta curioso observar que pese a haber encontrado el punto $P$ (correcto o no), buscan otro para dar la ecuación continua de la recta $s$ $(9,6 \%)$. Esto implica que no han acabado de comprender el problema.

Esta cuestión ha sido contestada por el 86,5\%; la puntuación máxima la ha obtenido el $5,5 \%$ y el $41,1 \%$ ha obtenido una calificación nula por no haber sabido hallar el punto de intersección entre el plano y la recta ni haber sabido hallar el vector director de la recta $s$ contenida el plano que además es perpendicular a $r$ y la corta. La puntuación media es de 0,57 y la desviación típica de 0,62. Esta cuestión ha sido aprobada por el $33,3 \%$ de los que la contestaron.

\section{Cuestión cuarta}

Para comprobar la igualdad propuesta en el primer apartado, los alumnos pueden proceder de varias maneras. No obstante, el 95,8\% de ellos ha calculado por separado ambos miembros de la igualdad para ver si coinciden. Un $4 \%$ ha decidido comprobar simplemente que, justificando que $A B=B A$ se verifica la igualdad siguiente: $(A+B)(A-B)=A 2-A B$ $+A B-B 2=A 2-B 2$.

El gran número de aprobados de esta cuestión se ha debido gracias a que el primer apartado de cálculo matricial ha ido bien pues valía la mitad de la cuestión. Ha habido dos aspectos a destacar en las resoluciones del primer apartado:

- Errores ingenuos cometidos de cálculo aritmético de números enteros al sumar, restar o multiplicar matrices $(13,6 \%)$

- Definiciones inventadas e incorrectas de la multiplicación de matrices $(12,7 \%)$

Las razones que han motivado el error en el segundo apartado han sido:

- Porque es una identidad notable que ya se verifica en los números reales, y además, para el primer apartado funciona $(25,4 \%)$

- Porque al ser cuadradas siempre se cumplirá que el número de columnas de la primera matriz que se multiplica por otra coincidirá con el número de filas de la segunda $(7,6 \%)$ 
La resolución de problemas en la prueba de Matemáticas de acceso a la universidad: procesos y errores

Alberto Mallart Solaz

Esta cuestión ha sido contestada por el 96,2\%; ha obtenido la puntuación máxima el $18 \%$ y el $4 \%$ ha obtenido una calificación nula por no haber sabido calcular el producto de matrices $\left(\mathrm{ni}(\mathrm{A}+\mathrm{B}) \cdot(\mathrm{A}-\mathrm{B}), \mathrm{ni}^{2} \mathrm{~A}^{2}-\mathrm{B}^{2}\right)$, ni tampoco haber sabido que la propiedad conmutativa no se verifica en el producto de matrices. La puntuación media es de 1,11 y la desviación típica de 0,55. Esta cuestión ha sido aprobada por el 78\% de los que la contestaron.

\section{Cuestión quinta}

El cálculo de la altura del triángulo equilátero presentado puede resolverse utilizando trigonometría $\left(\sin 60^{\circ}=\mathrm{h} / 8\right.$ ) o aplicando el teorema de Pitágoras a cualquiera de los dos triángulos rectángulos en que divide la altura al triángulo equilátero $\left(h=\sqrt{8^{2}-4^{2}}\right)$. Tanto se haya elegido la vía de la trigonometría (9,3\%) como la de Pitágoras $(69,8 \%)$, la altura es de $4 \sqrt{3}$.

La función a minimizar es la que resulta de sumar los cuadrados de las 3 distancias anteriores $\left(f(x)=3 x^{2}-8 \sqrt{3} x+80\right)$, y para encontrar el mínimo se debe igualar la derivada a $0(6 x-8 \sqrt{3}=0)$. Su resolución proporciona el valor de $x(x=4 \sqrt{3} / 3)$. Para comprobar que efectivamente se trata de un mínimo se puede calcular la segunda derivada $(6>0)$ o se pueden estudiar los intervalos de crecimiento de la primera derivada. Esta comprobación no era obligatoria aunque la han hecho el 23,3\%. Obsérvese que si no se ha resuelto correctamente el segundo apartado, el tercer apartado no puede hacerse.

Los errores recogidos para el cálculo de la altura del triángulo han consistido en no haber sabido redondear correctamente el valor de $4 \sqrt{3}$ o haber redondeado excesivamente al entero 7 perdiendo precisión (14\%). De hecho, el valor exacto sólo lo consideran el 34,9\%, el resto trabaja con una aproximación. Para el cálculo de las distancias del punto $P$ a los vértices $A, B, C$ se ha observado que hay alumnos que piensan que las distancias son las mismas o no saben calcular la distancia de $P$ a $B(11,6 \%)$.

Existen dos tipos de errores que aunque no sean generalizados, conviene señalar por su gravedad:

- Decálculo con raíces cuadradas: $\sqrt{x^{2}+16}=x+4 ;(\sqrt{48}-x)^{2}=48-x^{2}$

- Teóricos sobre el teorema de Pitágoras: 


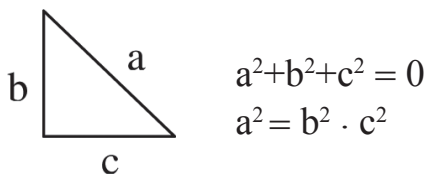

Esta cuestión ha sido contestada por el 34,6\%; la puntuación máxima la ha obtenido el $8,3 \%$ y el $16,7 \%$ ha obtenido una calificación nula por no haber sabido ni encontrar la altura del triángulo, ni haber sabido encontrar las distancias entre el punto $P$ y cada vértice. La puntuación media es de 0,82 y la desviación típica de 0,61. Esta cuestión ha sido aprobada por el $44,4 \%$ de los que la contestaron.

\section{Cuestión sexta}

Hay diferentes maneras de encontrar la ecuación del plano que contiene a $P, Q, R$. Una primera manera es multiplicar vectorialmente dos vectores linealmente independientes formados por estos 3 puntos para obtener el vector normal del plano buscado: $\overrightarrow{P Q} \times \overrightarrow{P R}=(6,3,2)$. Después, imponiendo el paso por uno de los puntos, se obtiene la $D(D=-6)$ consiguiendo la ecuación del plano: $6 x+3 y+2 z-6=0$ (escogida por un $17,8 \%)$. Una segunda manera es resolviendo un sistema de 3 ecuaciones con 4 incógnitas $(A, B, C, D)$ que resulta de imponer que $P, Q, R$ satisfagan $A x+B y+C z+D=0$ (escogida por un 2,5\%). Una tercera manera consiste en igualar a 0 el determinante formado por dos vectores linealmente independientes a partir de los tres puntos y el vector que tiene como origen cualquiera de los tres puntos y como final el punto de coordenadas generales $(x, y, z)$ (escogida por un 60,2\%). La comprobación de que $P, Q, R$ pertenecen al plano encontrado no era obligatoria, pero la han efectuado el $19,5 \%$.

Para contestar al segundo apartado viendo que los 4 puntos no son coplanarios, lo único que se debe hacer es substituir en la ecuación encontrada del plano las coordenadas del punto y comprobar que no se verifica $(33,1 \%)$. También se puede contestar este segundo apartado calculando el volumen del posible tetraedro que tiene por vértices los 4 puntos y viendo que no se anula o, equivalentemente, comprobando si se pueden conseguir tres vectores linealmente independientes partiendo de $P, Q, R, S(33,9 \%)$. 
La resolución de problemas en la prueba de Matemáticas de acceso a la universidad: procesos y errores

Alberto Mallart Solaz

Los alumnos han cometido errores de muy diversa índole en esta cuestión. A continuación se destacan dos tipos:

- Uso de argumentos teóricos erróneos para calcular el plano que contiene los tres puntos (11,9\%). Presentamos los dos argumentos mayoritarios:

$$
\left(\begin{array}{lll}
1 & 0 & 0 \\
0 & 2 & 0 \\
0 & 0 & 3
\end{array}\right) \Rightarrow x+2 y+3 z+D=0 ;\left|\begin{array}{ccc}
x-1 & y-0 & z-0 \\
0 & 2 & 0 \\
0 & 0 & 3
\end{array}\right|=0 \Rightarrow 6 x-6=0
$$

- Falta de coherencia en las resoluciones para ver si los 4 puntos son coplanarios $(8,5 \%)$, substituyendo las coordenadas de los puntos $P, Q, R$ en el plano que supuestamente generan y no satisfaciendo la ecuación.

Esta cuestión ha sido contestada por un 89,4\%; la puntuación máxima la ha obtenido el $44,1 \%$ y el $16,1 \%$ ha obtenido una calificación nula por no haber sabido calcular ninguna ecuación del plano que contiene los puntos $P, Q, R$ dados, ni tampoco haber sabido comprobar si el punto $S$ está en ese plano (un método adecuado era suficiente para anotar, a pesar de que el plano anterior se hubiera calculado mal). La puntuación media es de 1,34 y la desviación típica de 0,76. Esta cuestión ha sido aprobada por el $76,3 \%$ de los que la contestaron.

\section{Conclusiones y sugerencias}

La extracción de conclusiones representativas en la resolución de problemas es una tarea complicada pues requiere la implicación máxima del sujeto. Aprovechando la celebración de las PAU en dónde el alumno se juega el futuro, la voluntad está ciertamente asegurada.

La resolución depende obviamente del individuo pues intervienen a parte del conocimiento procedimental, factores como la predisposición a la tarea, la ansiedad y los prejuicios (Gómez-Chacón, 2000). Con el fin de reducir la ansiedad, los alumnos prefieren utilizar métodos rutinarios y mecánicos de resolución que aventurarse y probar nuevos caminos. Así, prefieren contestar cuestiones porque conocen el procedimiento que reflexionar sobre otras cuestiones (Nortes y otros, 2010). A continuación se resumen las características principales de cada cuestión 
mostrando un cuadro que expone el porcentaje de estudiantes que las ha elegido, que las ha aprobado, que ha aprendido la parte mecánica de su resolución y que ha sabido interpretar adecuadamente los resultados a los que ha llegado.

Habitualmente aparece en la prueba una pregunta sobre discusión de sistemas como la primera cuestión. Los argumentos teóricos que relacionan el álgebra con la posición relativa de rectas y planos no se han asimilado. El procedimiento mecánico común en todas las discusiones sí se ha aprendido. Así es que sería interesante en el aula subrayar la atención sobre la interpretación geométrica de los resultados.

\section{Tabla 5. Cuestión $1 .^{\mathrm{a}}$}

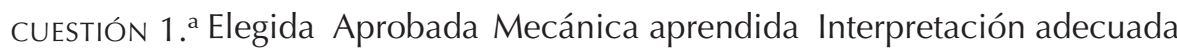

$$
81.7 \% \quad 36.5 \% \quad 82 \% \quad 64.1 \%
$$

En esta prueba de matemáticas la derivación se ha abordado desde un punto de vista geométrico en la segunda cuestión. La resolución es trivial y prácticamente inmediata. No obstante, la mitad no ha querido resolverla o no la ha entendido. Sería aconsejable enfatizar en el aula el sentido geométrico de las derivadas y el uso del lenguaje correspondiente.

\section{Tabla 6. Cuestión 2. ${ }^{\mathrm{a}}$}

CUESTIÓN 2. ${ }^{a}$ Elegida Aprobada Mecánica aprendida Interpretación adecuada $\begin{array}{llll}57.7 \% & 60 \% & 90.9 \% & 70.9 \%\end{array}$

La tercera cuestión ha sido una de las que más alumnos han decidido contestar pero es la que registra con diferencia más puntuaciones nulas $(41,1 \%)$ y argumentos teóricos inventados (51,3\%). Pero, ¿cómo es posible que el $35 \%$ no sepa calcular el vector director de la recta $r$ facilitada en el enunciado? Sería conveniente un trabajo en el aula que insistiera en la interpretación geométrica del álgebra.

\section{Tabla 7. Cuestión 3.}

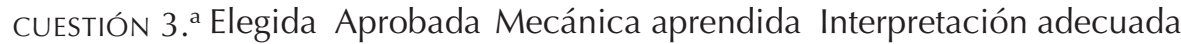

$$
86.5 \% \quad 33.3 \% \quad 67 \% \quad 48.7 \%
$$


La resolución de problemas en la prueba de Matemáticas de acceso a la universidad: procesos y errores

Alberto Mallart Solaz

La cuestión número 4 trata del cálculo matricial. Los resultados han sido muy positivos pues siendo la cuestión que más han contestado, también ha sido la que más han aprobado y la que menos puntuaciones nulas recoge $(4 \%)$. Es conveniente insistir en el aula no tanto sobre las similitudes entre las operaciones entre números reales y matrices, y más en sus diferencias. Les resulta incómodo pensar que la propiedad conmutativa no se verifique siempre; es prácticamente la primera ocasión en que se les expone una excepción.

Tabla 8. Cuestión $4 .^{a}$

CUESTIÓN 4. a Elegida Aprobada Mecánica aprendida Interpretación adecuada

$$
\begin{array}{llll}
96.2 \% & 78 \% & 87.3 \% & 66.7 \%
\end{array}
$$

Preocupa observar como la quinta cuestión ha sido la menos contestada. Para aprobarla bastan conocimientos que los alumnos ya han adquirido desde hace años (trigonometría o teorema de Pitágoras). Aún existen alumnos que no saben qué dice el teorema de Pitágoras ni cómo se utiliza. Otro error muy grave detectado ha sido el no saber operar con raíces cuadradas. Por otro lado, conviene reflexionar sobre la necesidad que tienen de aproximar el valor exacto de una raíz cuadrada perdiendo precisión. Un valor importante que la asignatura de Matemáticas debe inculcar (Decreto 143/2007 de 26 de junio; Decreto 142/2008, de 15 de julio) es la preocupación por la exactitud y precisión. Este hecho suscita nuevamente el tema del mal uso de las calculadoras. En el aula se debe seguir insistiendo en que la calculadora ayuda pero no substituye; debe supervisar los resultados el alumno con un espíritu crítico y preciso.

Tabla 9. Cuestión 5. ${ }^{\text {a }}$

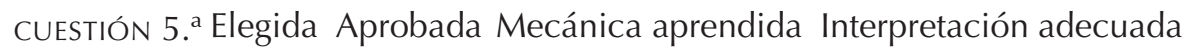

$$
\begin{array}{llll}
34.63 \% & 44.4 \% & 70.1 \% & 83.3 \%
\end{array}
$$

La última cuestión ha sido una de las más respondidas y se han detectado errores ocasionados por una mala asimilación de los argumentos teóricos por un lado, y de coherencia y falta de espíritu crítico por otro lado. En Matemáticas se debe impartir el valor de la coherencia en las cadenas de argumentos lógicos. Por ello en el aula, además de trabajar los problemas propios de la geometría afín analizando sus partes exhaustivamente, también conviene trabajar la comprensión global (Mallart y Deulofeu, 2012) y el sentido que cada parte tiene dentro de la resolución total. 
La resolución de problemas en la prueba de Matemáticas de acceso a la universidad:

procesos y errores

Alberto Mallart Solaz

\section{Tabla 10. Cuestión 6. ${ }^{\mathrm{a}}$}

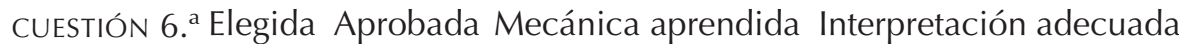

$$
\begin{array}{llll}
89.4 \% & 76.3 \% & 80.5 \% & 79.6 \%
\end{array}
$$

En conclusión, se han observado 5 puntos comunes en todas las resoluciones sobre los que se puede reflexionar y abordar en el aula:

1. Correcta asimilación de los procedimientos mecánicos de resolución

2. Incapacidad de resolver creativamente cuestiones sobre la interpretación geométrica del álgebra

3. Incorrecta asimilación de las operaciones y propiedades del álgebra matricial

4. Indiferencia frente a resultados parciales incoherentes y contradictorios sobre los que construyen su propia argumentación

5. Ausencia de rigor y precisión en los cálculos efectuados

Los estudios que se han tomado como referencia en la misma línea de analizar muestras de exámenes de las PAU para proporcionar un conocimiento de los errores más frecuentes y sugerir alternativas para ayudar a combatirlos han sido: Boal y otros (2008), Miralles y otros (2009), Nortes y otros (2010), Ruiz de Gauna y otros (2011).

Boal y otros (2008) afirman que existe poca penalización de la ortografía matemática en las PAU y que hay poca presencia de preguntas que requieren y valoran la comunicación matemática. En el presente estudio se han comprobado tales observaciones y se ha tratado de ir más lejos al analizar la falta de rigor y precisión en los cálculos efectuados por un lado, y por otro la interpretación adecuada frente a resultados incoherentes y contradictorios en la propia argumentación (aspecto vital en las cadenas lógicas que resulta inadmisible y preocupante en la Cuestión 3. ${ }^{\mathrm{a}}$ con $48.7 \%$ ).

Miralles y otros (2009) afirman que la elección de unas cuestiones u otras en las PAU se ve influenciada por el tipo de resolución requerida: ya sea porque se deben relacionar diversos apartados, o porque exige recursos operativos sofisticados, o porque admite diversas resoluciones en función del razonamiento utilizado. En este estudio se ha querido comprobar y profundizar sobre el tipo de resoluciones de los alumnos. Por este motivo se han analizado qué soluciones han sido de tipo mecánicas y rutinarias y cuáles han sido más creativas. Se ha observado que 
La resolución de problemas en la prueba de Matemáticas de acceso a la universidad: procesos y errores

Alberto Mallart Solaz

los aspectos mecanicistas de las resoluciones se han sabido reproducir correctamente. No obstante, a la hora de resolver cuestiones creativamente sobre la interpretación geométrica del álgebra se han observado carencias.

Nortes y otros (2010) analizan respuestas y errores en las cuestiones y afirman que los alumnos no varían un planteamiento sin salida, e incluso reiteran un procedimiento conocido sin poder pensar en otro. De hecho, afirman que los estudiantes prefieren contestar una cuestión de resolución conocida que reflexionar sobre otra cuestión aunque resulte de resolución más inmediata. Siguiendo la misma línea, esta investigación corrobora que a pesar de conocer las partes mecánicas de las resoluciones de las cuestiones, éstas no se consiguen aprobar (Cuestión 1. a, Cuestión $3 .^{a}$ y Cuestión $5 .^{a}$ ). Se puede observar como los resultados no guardan relación con la elección de lo que saben mejor, como dice Miralles y otros (2009). Se podría afirmar que la elección guarda relación con las cuestiones que tienen una resolución mecánica conocida.

Ruiz de Gauna y otros (2011) analizan problemas de las PAU (desde el 1994 hasta el 2008) subrayando la existencia de problemas-tipo análogos en su método de resolución y que muchos de ellos se trabajan en las aulas. Afirma que así, el estilo de la prueba influye en la metodología de enseñanza, en la elaboración de libros de texto y en los estilos de enseñanza de los profesores. En este estudio se ha ido más allá analizando y comprobando cómo las partes mecánicas de las resoluciones se tienen bien asimiladas.

El estudio de la resolución de problemas en las PAU brinda una aproximación a los errores que cometen los alumnos cuando intentan solucionar con voluntad e interés una situación. Con este trabajo se quiere contribuir a la detección de errores y ayudar subrayando aspectos susceptibles de mejora en la enseñanza y aprendizaje de las Matemáticas en el nivel preuniversitario.

El hecho de haber comprobado una asimilación correcta en general sobre la parte mecánica de las resoluciones pero al mismo tiempo haber verificado una ausencia de rigor y de precisión en las operaciones plantea nuevos interrogantes. Una línea de investigación podría preocuparse de investigar la relativización de la precisión frente a la importancia del proceso resolutivo. Otra línea de investigación podría tratar las causas que originan la indiferencia ante errores de incoherencia en la propia argumentación. Probablemente subsanar un aspecto ayudaría a mejorar el otro. 
La resolución de problemas en la prueba de Matemáticas de acceso a la universidad:

procesos y errores

Alberto Mallart Solaz

\section{Referencias bibliográficas}

Boal, N.; Bueno, C.; Lerís, L. y Sein-Echaluce, M.L. (2008). Las habilidades matemáticas evaluadas en las pruebas de acceso a la Universidad: un estudio en varias universidades públicas españolas. Revista de Investigación Educativa, RIE,26,1,11-23.

Corominas, E. (2001). La transición a los estudios universitarios. Abandono o cambio en el primer año de universidad. Revista de Investigación Educativa, 19, 1, 127-151.

Departament d'Educació (2007). Decreto 143/2007, de 26 de junio, por el cual se establece la ordenación de las enseñanzas de Educación Secundaria Obligatoria en Cataluña (DOGC 4915, de 29-06-2007).

Departament d'Educació (2008). Decreto 142/2008, de 15 de julio, por el cual se establece la ordenación de las enseñanzas de Bachillerato en Cataluña (DOGC 5183, de 29-07-2008).

Gómez-Chacón, I.M. (2000). Matemática emocional. Los afectos en el aprendizaje matemático. Madrid: Narcea. Mates para aprender a pensar mejor, p. 7.

Instituto de Evaluación (2010). Resultados en España del estudio PISA 2009. Madrid: Ministerio de Educación.

Mallart, A. y Deulofeu, J. (2012). Una estrategia para mejorar la comprensión de los enunciados de los problemas. Revista de didáctica de las Matemáticas, UNO, 59, 83-92.

Miralles, I. y Deulofeu, J. (2009). Dificultad subjetiva de la prueba de Matemáticas de las PAU. ¿Qué eligen los alumnos? XIV Jornadas de Aprendizaje y Enseñanza de las Matemáticas. Girona, España, 1 al 4 de julio.

Nortes, A. y Nortes, R. (2010). Resolución de problemas de matemáticas en las pruebas de acceso a la Universidad: errores significativos. Educatio siglo XXI, 28, 1, 317-342.

OCDE (2006). PISA 2006. Marco de evaluación. Conocimientos y habilidades en Ciencias, Matemáticas y Lectura. Madrid: Santillana; MEC.

Riaguas, A.; Arribas, M.; Celorrio, R. y Lerís, D. (2006). El acceso a los estudios de Ingeniería: detección de debilidades o carencias formativas en Matemáticas. Actas del $4^{\circ}$ Congreso Internacional de Docencia Universitaria e Innovación, CIDUI. Barcelona, España, 5 al 7 de julio.

Rico, L. (2007). La competencia matemática en PISA. PNA, 1(2), 47-66.

Ruiz de Gauna, J.; Sarasua, J. y García Iturrioz, J.M. (2011). Una tipología y clasificación de los ejercicios de matemáticas de selectividad. Revista de Educación Matemática, Epsilón, 28(2), 78, 21-38. 
